\title{
The anti-atherogenic effects of berberine on foam cell formation are mediated through the upregulation of sirtuin 1
}

\author{
LIYI CHI ${ }^{1}$, LIJING PENG $^{2}$, NA PAN $^{1}$, XIAOJING HU $^{4}$ and YANHAI ZHANG ${ }^{3}$ \\ Departments of ${ }^{1}$ Neurology, ${ }^{2}$ Cardiovascular Medicine and ${ }^{3}$ Cadres Ward, The 451st Hospital of People's Liberation Army; \\ ${ }^{4}$ Department of Cardiovascular Medicine, Ninth Hospital of Xi'an, Xi'an, Shaanxi 710054, P.R. China
}

Received February 22, 2014; Accepted July 2, 2014

DOI: $10.3892 /$ ijmm.2014.1868

\begin{abstract}
Berberine (BBR) is a botanical alkaloid that has been reported to have effects in cardiovascular diseases; however, the mechanisms involved are not yet fully understood. In the present study, the protective effects of BBR were evaluated, and the underlying molecular mechanisms were investigated. The effects of a combination of atorvastatin and BBR on foam cell formation were also investigated. THP-1-derived macrophages were pre-treated with BBR $(5,10$ and $20 \mathrm{mg} / \mathrm{l})$ for $2 \mathrm{~h}$ prior to the addition of oxidized low density lipoprotein (ox-LDL; $50 \mathrm{mg} / \mathrm{l}$ ). Small interfering RNA (siRNA) targeting sirtuin 1 (SIRT1) and the adenosine 5'-monophosphate-activated protein kinase (AMPK) inhibitor, compound $\mathrm{C}$, were used to investigate the mechanisms through which BBR exerts its effects. To determine the effect of a combination of atorvastatin and $\mathrm{BBR}$, the macrophages were treated with atorvastatin and BBR separately or jointly for $2 \mathrm{~h}$, and then treated with ox-LDL $(50 \mathrm{mg} / \mathrm{l})$ or lipopolysaccharide (LPS; $10 \mu \mathrm{M})$ for $12 \mathrm{~h}$. Oil Red O staining was used to detect foam cell formation. Lipid amounts were assessed by high-performance liquid chromatography (HPLC). Gene and protein expression was evaluated by RT-qPCR, western blot analysis and enzyme-linked immunosorbent assay (ELISA) carried out separately or jointly. The results from Oil Red O staining and HPLC revealed that BBR effectively suppressed foam cell formation and lipid and cholesterol accumulation. Furthermore, BBR upregulated the expression of SIRT1 and AMPK and downregulated the expression of peroxisome proliferator-activated receptor- $\gamma($ PPAR- $\gamma)$. Pre-treatment of the cells with SIRT1-siRNA or compound C attenuated the anti-atherosclerotic effects of BBR. The results obtained in the present study demonstrate that the combination of atorvastatin and BBR is more effective in inhibiting foam cell formation than using atorvastatin alone. These data suggest that BBR suppresses foam cell formation by activating the
\end{abstract}

Correspondence to: Dr Yanhai Zhang, Department of Cadres Ward, The 451st Hospital of People's Liberation Army, No. 269 Youyi Road, Beilin, Xi'an, Shaanxi 710054, P.R. China

E-mail: yanhaizhangxian@163.com

Key words: atherosclerosis, THP-1 cells, foam cell formation, berberine, sirtuin 1, inflammation, lipid metabolism
AMPK-SIRT1-PPAR- $\gamma$ pathway and diminishing the uptake of ox-LDL. Combination therapy with BBR and atorvastatin was more effective in preventing atherosclerotic processes than atorvastatin alone.

\section{Introduction}

Atherosclerosis, a progressive disease characterized by excessive cholesterol deposition and persistent inflammation within the artery wall. Epidemiological and experimental evidence indicates that the deregulation of cholesterol metabolism is the most important risk factor for the development of atherosclerosis (1). Monocytes play an important role in the progression of the disease $(2,3)$. Modified lipoproteins recruit monocytes to the vascular intima, where monocytes differentiate into macrophages to engulf these lipoprotein molecules and then become foam cells $(4,5)$. The accumulation of macrophagederived foam cells in the subendothelial space is the crucial step in the initiation and progression of atherosclerosis (6).

The balance between lipids entering into and moving out of the macrophage is necessary to avoid lipid overload, and ultimately, atheroma development (7). Silent information regulator T1 (sirtuin 1 or SIRT1), a NAD ${ }^{+}$-dependent histone deacetylase, is a fundamental factor in sensing caloric restriction, improving insulin secretion in pancreatic $\beta$ cells, and reducing the accumulation of fatty acids in white adipose tissue (8). SIRT1 has various targets, including nuclear peroxisome proliferator-activated receptor- $\gamma$ (PPAR- $\gamma$ ), PPAR- $\gamma$ activator $1 \alpha$ (PGC- $1 \alpha)$ and p53, many of which have also been shown to play a role in atherogenesis $(9,10)$; however, little is known about the association between SIRT1 and atherosclerosis. In atherogenesis, SIRT1 overexpression has been shown to prevent atherosclerosis by improving vascular function (11). In particular, the role of SIRT1 in monocyte adhesion, macrophage infiltration, lipid uptake and foam cell formation remains to be determined.

Berberine (BBR) is a botanical alkaloid isolated from traditional Chinese medicinal herbs, such as Coptidis Rhizoma (Huanglian) and Cortex Phellodendri (Huangbai) (12). BBR is known to have protective effects in cardiovascular diseases. For example, BBR decreases plasma cholesterol and glucose levels $(13,14)$. It has been used as an anti-inflammatory agent, antimicrobial agent, antihypertensive agent, anti-arrhythmic agent, antitumor agent and an antidiabetic agent (15). In addition, 
BBR exerts antioxidant activity by upregulating the expression of the cellular survival-associated factor, SIRT1 (16). However, to the best of our knowledge, the effects of BBR on macrophage foam cell formation and intracellular cholesterol metabolism remain unexplored.

In the present study, we aimed to investigate the molecular mechanisms underlying the anti-atherogenic effects of BBR on foam cell formation and the role of SIRT1 in these processes.

\section{Materials and methods}

Cell culture. The human monocytic cells, THP-1 (ATCC, Rockville, MD, USA), were maintained in RPMI-1640 medium containing $10 \%$ fetal bovine serum (FBS), $0.05 \mathrm{mM} 2$-mercaptoethanol, $10 \mathrm{mM}$ HEPES, $1 \mathrm{mM}$ sodium pyruvate, $4.5 \mathrm{~g} / 1$ glucose and $1.5 \mathrm{~g} / \mathrm{l}$ bicarbonate in a humidified atmosphere of $5 \% \mathrm{CO}_{2}$ and $95 \%$ air at $37^{\circ} \mathrm{C}$. The differentiation of THP-1 monocytes into macrophages was induced by exposure to $100 \mathrm{nM}$ phorbol 12-myristate 13-acetate (PMA) (Sigma-Aldrich, St. Louis, MO, USA) for $48 \mathrm{~h}$. The differentiated THP-1 macrophages were extensively washed in PBS before being used in the experiments.

Extraction and oxidation of low density lipoprotein $(L D L)$. The extraction and oxidation of LDL were performed according to a previously performed method (17). For the production of oxidized LDL (ox-LDL), $\mathrm{CuSO}_{4}$ was added to $\mathrm{LDL}$ at a final concentration of $10 \mu \mathrm{M}$ for $24 \mathrm{~h}$ at $37^{\circ} \mathrm{C}$ and the mixture was then dialyzed for $24 \mathrm{~h}$ at $4^{\circ} \mathrm{C}$. Finally, the bacteria in the solution were removed through ultrafiltration.

Treatment with BBR. The THP-1-derived macrophages were divided into an ox-LDL (50 mg/l) group and BBR (5, 10 and 20 mg/l; Wuhan Fortuna Chemical Co., Ltd., Wuhan, China) groups. The experiments were performed in serum-free (SF) experimental medium. The BBR groups were co-cultured with BBR for $2 \mathrm{~h}$ prior to the addition of ox-LDL, which was added at a concentration of $50 \mathrm{mg} / \mathrm{l}$. These cells were then collected after having been incubated for $24 \mathrm{~h}$.

Transfection with small interfering RNA (siRNA). The transient transfection of siRNA into THP-1-derived macrophages was performed using Lipofectamine RNAi MAX (Sigma-Aldrich). The oligos used for SIRT1-siRNA have been previously described (9). The negative control (NC) group was transfected with a siRNA sequence which had no effect on gene expression. Subsequently, the cells were exposed to BBR $(10 \mathrm{mg} / \mathrm{l})$ with or without ox-LDL (50 mg/l) for $12 \mathrm{~h}$.

Treatment with inhibitor. Compound C (Sigma-Aldrich) is a specific adenosine 5'-monophosphate (AMP)-activated protein kinase (AMPK) inhibitor. Compound C (10 $\mu \mathrm{M}$, in DMSO) was added $15 \mathrm{~min}$ prior to treatment with BBR. For treatment, the cells were treated only with BBR $(10 \mathrm{mg} / \mathrm{l})$ for $24 \mathrm{~h}$ or BBR was added to the cultures to a final concentration of $10 \mathrm{mg} / \mathrm{l}$ for $2 \mathrm{~h}$ prior to the addition of ox-LDL, which was added at a concentration of $50 \mathrm{mg} / \mathrm{l}$. These cells were then collected after $12 \mathrm{~h}$ of incubation.

Treatment with BBR and atorvastatin. The THP-1-derived macrophages were divided into atorvastatin (20 and $40 \mu \mathrm{M}$; SigmaAldrich), BBR (10 mg/l) and atorvastatin plus BBR (atorvastatin
Table I. Oligonucleotides used in RT-qPCR.

Genes Primers

$\beta$-actin Forward: 5'-GATCATTGCTCCTCCTGAGC-3' Reverse: 5'-ACTCCTGCTTGCTGATCCAC-3'

SIRT1 Forward: 5'-GAGTGGCAAAGGAGCAGA-3' Reverse 5'-TCTGGCATGTCCCACTATC-3'

PPAR- $\gamma$ Forward: 5'-GCAGTGGGGATGTCTCATAATGC-3' Reverse: 5'-CAGGGGGGTGATGTGTTTGAA-3'

MCP-1 Forward: 5'-AGCCACCTTCATTCCCCAAG-3' Reverse: 5'-CTCCTTGGCCACAATGGTCT-3'

SIRT1, sirtuin 1; PPAR- $\gamma$, peroxisome proliferator-activated receptor- $\gamma$; $\mathrm{MCP}-1$, monocyte chemotactic protein-1.

$20 \mu \mathrm{M}$, BBR $10 \mathrm{mg} / \mathrm{l})$ groups. The experiments were performed in SF experimental medium. The treatment groups were co-cultured with the drugs for $2 \mathrm{~h}$ and were then treated with ox-LDL $(50 \mathrm{mg} / \mathrm{l})$ or lipopolysaccharide (LPS; $10 \mu \mathrm{M}$; Sigma-Aldrich) for $12 \mathrm{~h}$.

Foam cell formation assay and cytoplasmic lipid detection. The formation of foam cells was evaluated using Oil Red O staining. The THP-1-derived macrophages were cultured with ox-LDL for $24 \mathrm{~h}$ and then washed 3 times with PBS. Following fixation with $4 \%$ formaldehyde for $15 \mathrm{~min}$, the cells were stained with Oil Red O (3 mg/ml in 60\% isopropanol; SigmaAldrich) for $10 \mathrm{~min}$ at $37^{\circ} \mathrm{C}$ to evaluate the characteristic lipid accumulation in macrophage-derived foam cells. The cells were then rinsed with water, and hematoxylin was introduced to label the cell nuclei. Foam cell formation was observed under a microscope, and Oil Red O staining was assessed by a color density assay using iVision Software. The density of lipid content was evaluated by alcohol extraction after staining. The absorbance at $540 \mathrm{~nm}$ was measured using a microplate reader (BioTek Instruments, Winooski, VT, USA).

Lipid analysis by high-performance liquid chromatography $(H P L C)$. Cellular total triglyceride content and cholesterol levels were analyzed by HPLC. Briefly, the cells were rinsed in PBS 3 times and lysed by the addition of a $0.9 \% \mathrm{NaOH}$ solution. Masterol was used as a standard, and the samples were dissolved in $100 \mu \mathrm{l}$ of isopropanol-acetonitrile (v/v, 20:80), followed by incubation in ultrasound water at room temperature for $5 \mathrm{~min}$. Finally, the samples were placed in the Agilent 1100 series HPLC system (Agilent Technologies, Santa Clara, CA, USA).

Analysis of mRNA and protein expression. Total RNA was extracted using TRIzol (Sigma-Aldrich) reagent and reverse transcribed into cDNA. The cDNA was quantified by SYBRGreen qPCR using the SYBR Premix Ex Taq ${ }^{\mathrm{TM}}$ II kit (Takara Bio Inc., Otsu, Japan). The reaction conditions followed the instructions provided by the manufacturers. Gene-specific primers for monocyte chemotactic protein-1 (MCP-1), SIRT1, PPAR- $\gamma$ and $\beta$-actin (Table I) were used in the reactions.

Protein expression was evaluated by western blot analysis. Specific antibodies for phospho-AMPKa (Thr172), AMPK, 

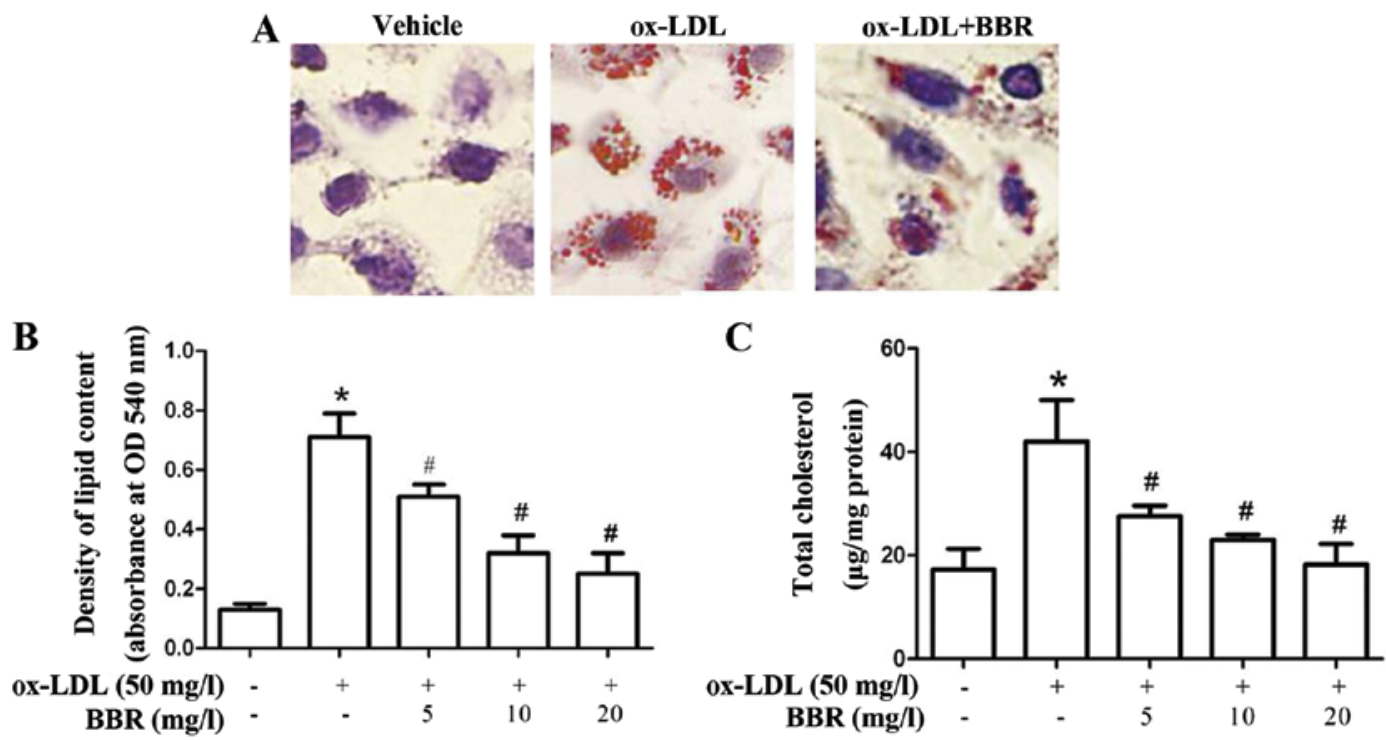

Figure 1. Berberine (BBR) attenuates foam cell formation and lipid accumulation in macrophages. THP-1-derived macrophages were pre-treated with BBR (5, 10 and $20 \mathrm{mg} / \mathrm{l}$ ) for $2 \mathrm{~h}$ and then treated with oxidized low density lipoprotein (ox-LDL; $50 \mathrm{mg} / \mathrm{l}$ ) for $24 \mathrm{~h}$. (A) Foam cell formation was analyzed through Oil Red O staining; (B) density of lipid content was evaluated by alcohol extraction, and absorbance was measured at $540 \mathrm{~nm}$; (C) cholesterol ester was assayed as described in Materials and methods. Data are the means \pm SD from 6 independent experiments. ${ }^{*} \mathrm{P}<0.05$ vs. control (untreated cells); ${ }^{*} \mathrm{P}<0.05$ vs. treatment with ox-LDL alone.

SIRT1, PPAR- $\gamma$ and $\beta$-actin were purchased from Cell Signaling Technology (Danvers, MA, USA) and Sigma-Aldrich. Protein concentrations were determined using the Bio-Rad protein assay kit (Bio-Rad Laboratories, Hercules, CA, USA). Equal amounts of protein $(20 \mu \mathrm{g})$ from each lysate were subjected to SDS-PAGE. The proteins were transferred onto nitrocellulose membranes at $80 \mathrm{~V}$ for $1 \mathrm{~h}$ and blocked for $4 \mathrm{~h}$ in $5 \%$ skim milk, then incubated overnight at $4^{\circ} \mathrm{C}$ with a 1:500 dilution of primary antibody (produced in rabbit and mouse) followed by incubation with the corresponding secondary antibody (goat anti-rabbit or anti-mouse $\operatorname{IgG}$ ). The enhanced chemiluminescence system (Amersham Pharmacia Biotech Inc., Piscataway, NJ, USA) was used for detection. Filters were subsequently exposed to Kodak BioMax light-1films (Eastman Kodak, Rochester, NY, USA) and the intensity of the western blot signals was quantified by densitometry.

Enzyme-linked immunosorbent assay (ELISA). To evaluate the levels of MCP-1 produced, the THP-1-derived macrophages were pre-treated with BBR or atorvastatin, or a combination of both for $2 \mathrm{~h}$ then treated with LPS for $12 \mathrm{~h}$. Supernatants from the treated cells were collected and analyzed for MCP-1 using a sandwich ELISA kit (R\&D Systems, Minneapolis, MN, USA) according to the manufacturer's instructions.

Statistical analysis. Data are presented as the means \pm standard deviation ( $\mathrm{SD}, \mathrm{n}=6)$. All data were evaluated using SPSS 11.0 software. Groups were compared using analysis of variance (ANOVA) followed by the Student's t-test. P-values $<0.05$ or $<0.01$ were considered to indicate statistically significant differences.

\section{Results}

BBR inhibits ox-LDL-induced foam cell formation and cholesterol accumulation in macrophages. To investigate the effects of BBR on ox-LDL-induced foam cell formation, the THP-1-derived macrophages were treated with ox-LDL in the presence or absence of BBR for $24 \mathrm{~h}$. BBR effectively suppressed ox-LDL-induced foam cell formation (Fig. 1A). The increase in lipid and cholesterol accumulation was significantly attenuated by treatment with BBR in a dose-dependent manner (Fig. 1B and C), which suggests that BBR abrogates the formation of foam cells by regulating lipid accumulation.

BBR inhibits lipid accumulation through SIRT1 and PPAR- $\gamma$. SIRT1 can reduce the accumulation of fatty acids by repressing PPAR- $\gamma$ (18). In this study, to explore the molecular mechanisms involved in the lipid lowering effects of BBR, the THP-1-derived macrophages were treated with ox-LDL in the presence or absence of BBR for $24 \mathrm{~h}$ and the effects of BBR on the expression of SIRT1 and PPAR- $\gamma$ were then examined. Treatment with BBR increased the expression of SIRT1 and decreased the expression of PPAR- $\gamma$ at both the protein and mRNA level in the macrophages in a dose-dependent manner (Fig. 2).

Inhibition of SIRT1 function abrogates the lipid-lowering effects of BBR. To further investigate the role of SIRT1 in the lipid-lowering effects of BBR, the THP-1-derived macrophages were transfected with SIRT1 siRNA in the presence of BBR. Pre-treatment of the macrophages with SIRT1 siRNA diminished the effects of BBR on the expression of SIRT1 and PPAR- $\gamma$ at both the protein and mRNA level (Fig. 3A and B). Additionally, pre-transfection with SIRT1 siRNA abolished the BBR-mediated suppression of oxLDL-induced lipid accumulation (Fig. 3C and D), suggesting that the induction of SIRT1 is required for BBR-mediated protection against foam cell formation.

Role of AMPK in the BBR-mediated increase in SIRTI expression. The activation of AMPK has been suggested to play an important role in SIRT1 gene expression and to switch off a number of processes that consume ATP, such as fatty acid, protein and cholesterol synthesis (19-22). In this study, 

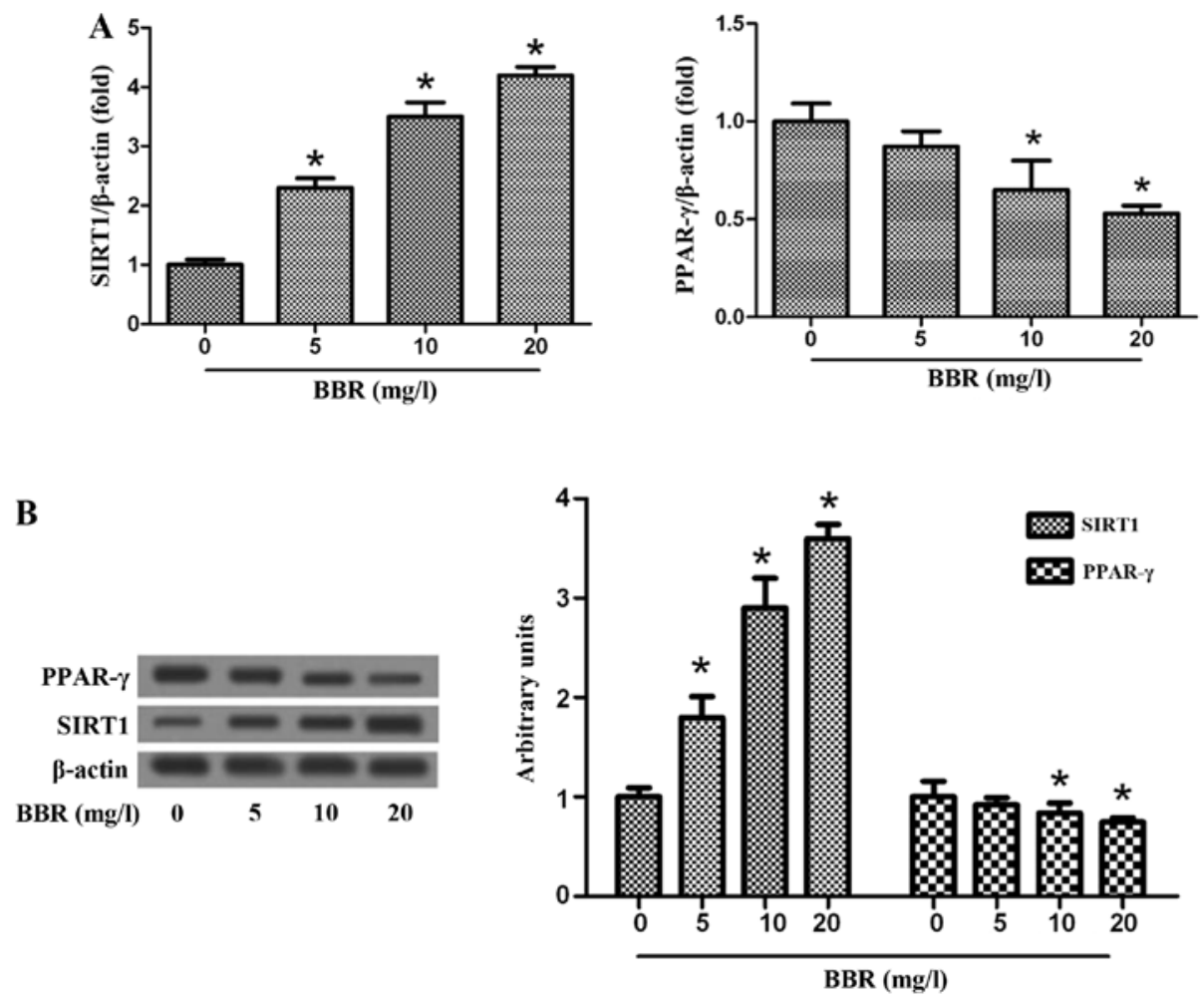

Figure 2. Effects of berberine (BBR) on the expression of sirtuin 1 (SIRT1) and peroxisome proliferator-activated receptor- $\gamma$ (PPAR- $\gamma$ ) in THP-1-derived macrophages. THP-1-derived macrophages were pre-treated with BBR (5, 10 and $20 \mathrm{mg} / \mathrm{l})$ for $24 \mathrm{~h}$. (A) mRNA levels of SIRT1 and PPAR- $\gamma$; (B) protein levels of SIRT1 and PPAR- $\gamma$. Data are the means \pm SD from 6 independent experiments. ${ }^{*}<<0.05$ vs. control (untreated cells).
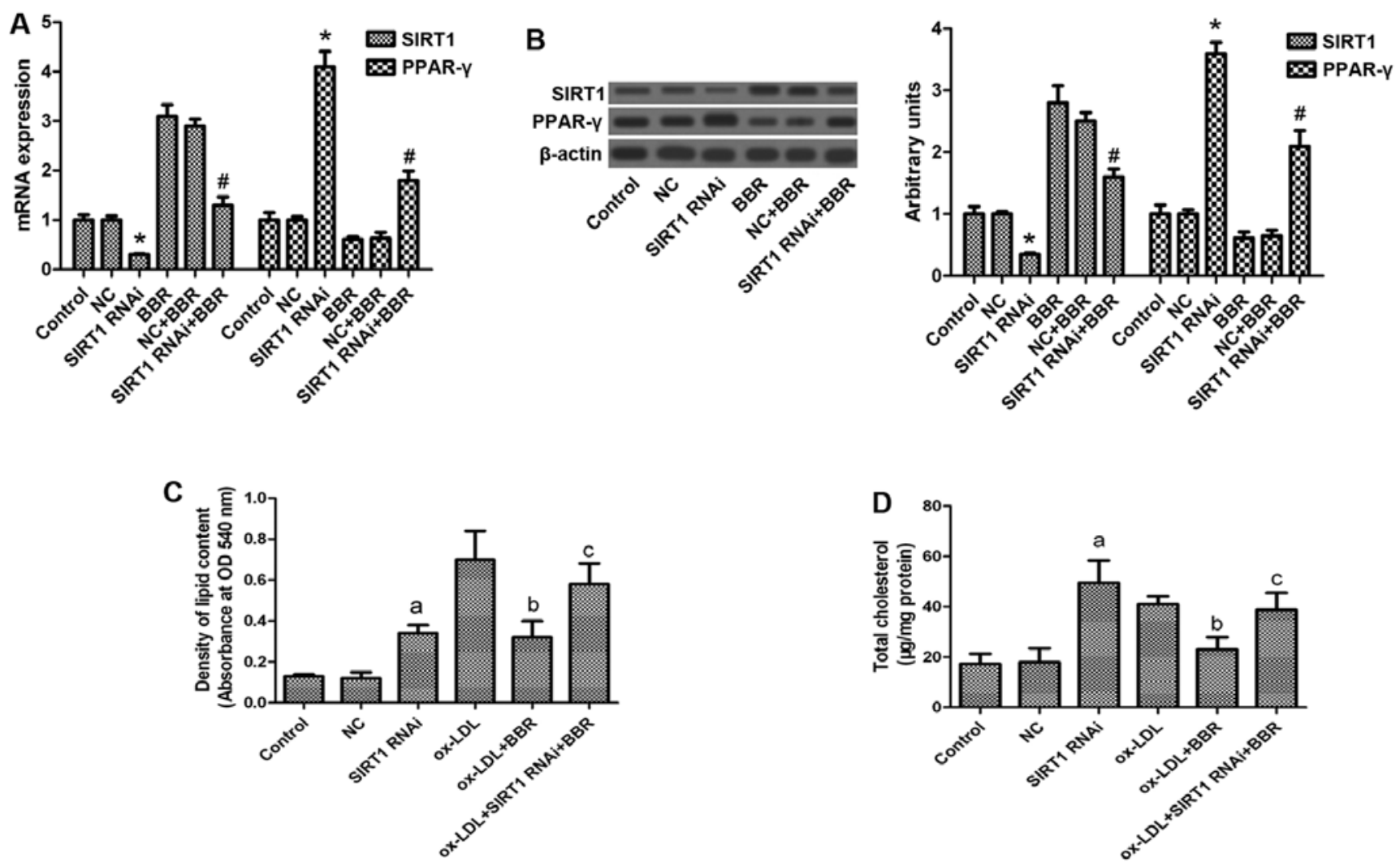

Figure 3. Inhibition of sirtuin 1 (SIRT1) function abrogates the lipid-lowering effects of berberine (BBR). THP-1-derived macrophages were pre-treated with SIRT1 siRNA, and were then treated with BBR $(10 \mathrm{mg} / \mathrm{l})$ for $24 \mathrm{~h}$ or were exposed to oxidized low density lipoprotein (ox-LDL; $50 \mathrm{mg} / \mathrm{l})$ for $12 \mathrm{~h}$ with or without BBR (10 mg/l) pre-treatment for $2 \mathrm{~h}$. (A) mRNA levels of SIRT1 and peroxisome proliferator-activated receptor- $\gamma$ (PPAR- $\gamma$ ); (B) protein levels of SIRT1 and PPAR- $\gamma$; (C) density of lipid content; (D) cholesterol ester was assayed by HPLC. Data are the means \pm SD from 6 independent experiments. "P<0.05 vs. control (untreated cells); ${ }^{*} \mathrm{P}<0.05$ vs. treatment with $\mathrm{BBR} ;{ }^{\mathrm{a}} \mathrm{P}<0.05$ vs. negative control $(\mathrm{NC}) ;{ }^{\mathrm{b}} \mathrm{P}<0.05$ vs. treatment with $\mathrm{ox}-\mathrm{LDL} ;{ }^{\mathrm{c}} \mathrm{P}<0.05$ vs. treatment with ox-LDL $+\mathrm{BBR}$. 
A
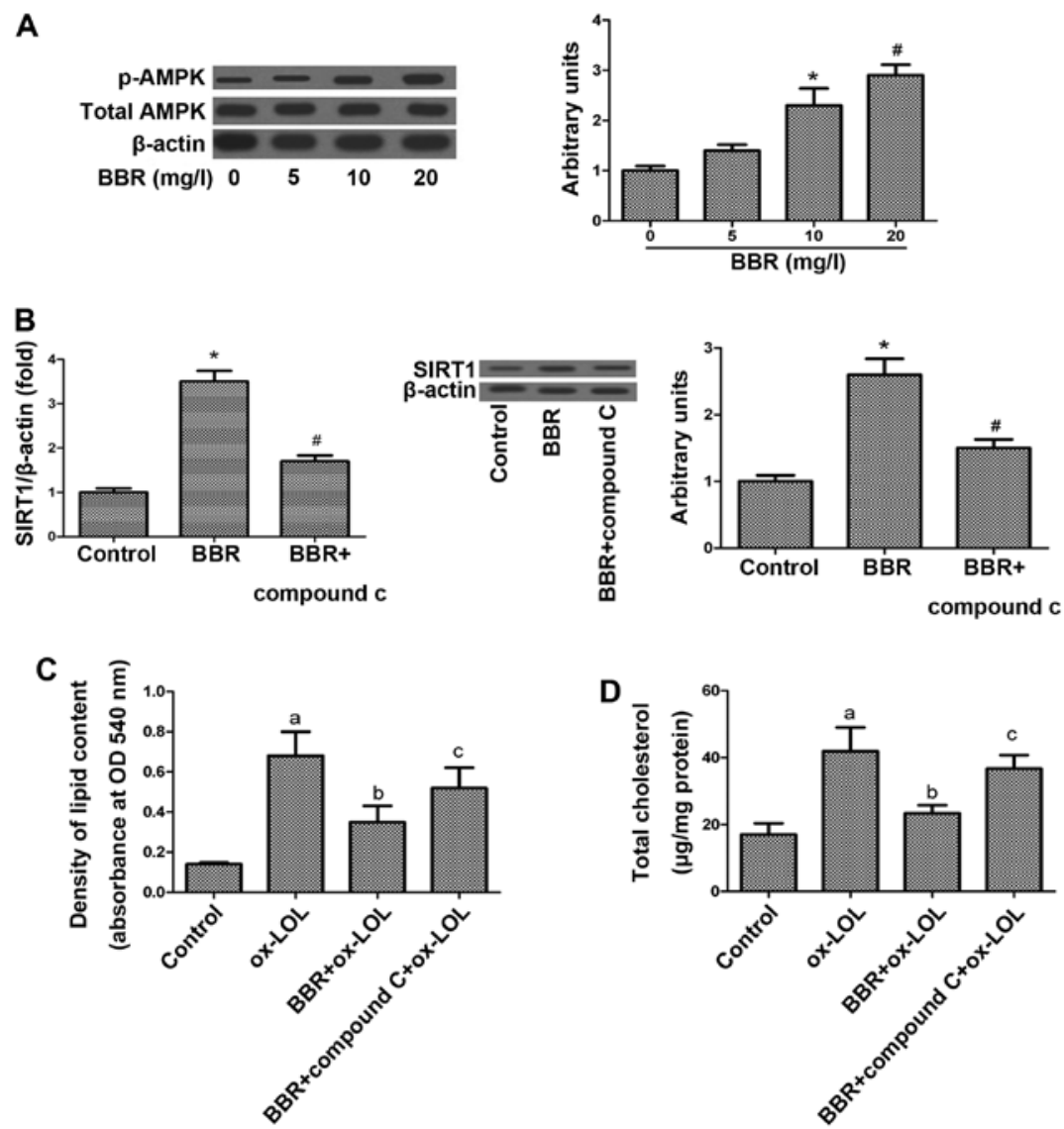

Figure 4. Role of adenosine 5'-monophosphate (AMP)-activated protein kinase (AMPK) in the berberine (BBR)-mediated increase in sirtuin 1 (SIRT1) expression. THP-1-derived macrophages were treated with BBR $(5,10$ and $20 \mathrm{mg} / \mathrm{l})$ for $24 \mathrm{~h}$. THP-1-derived macrophages were pre-treated with compound C (AMPK inhibitor, $10 \mu \mathrm{M}$ ) for $15 \mathrm{~min}$, and then treated with BBR (10 mg/l) for $24 \mathrm{~h}$ or were exposed to oxidized low density lipoprotein (ox-LDL; $50 \mathrm{mg} / \mathrm{l})$ for $12 \mathrm{~h}$ with or without BBR (10 mg/l) pre-treatment for $2 \mathrm{~h}$. (A) Activation of AMPK; (B) mRNA and protein levels of SIRT1; (C) density of lipid content; (D) cholesterol ester was assayed by HPLC. Data are the means \pm SD from 6 independent experiments. ${ }^{*} \mathrm{P}<0.05$ vs. control (untreated cells); $\mathrm{P}<0.05$ vs. treatment with BBR; ${ }^{\mathrm{a}} \mathrm{P}<0.05$ vs. control; ${ }^{\mathrm{b}} \mathrm{P}<0.05$ vs. treatment with ox-LDL; ${ }^{\mathrm{C}} \mathrm{P}<0.05$ vs. treatment with ox-LDL $+\mathrm{BBR}$.

to determine whether AMPK is involved in the BBR-induced upregulation of SIRT1, we examined the expression of AMPK in response to BBR. The phosphorylated isoform is the active AMPK form; thus, we determined the phosphorylated AMPK/ total AMPK protein ratio. BBR significantly increased the phosphorylation of AMPK (Fig. 4A) suggesting that AMPK may contribute to the enhancing effects of BBR on SIRT1 expression in macrophages.

To verify this hypothesis, the THP-1-derived macrophages were pre-treated with an AMPK inhibitor (compound C). Pre-treatment of the macrophages with compound $\mathrm{C}$ diminished the BBR-induced expression of SIRT1 at both the protein and mRNA level (Fig. 4B). In addition, the suppression of lipid accumulation induced by BBR was reversed when the macrophages were pre-treated with compound C (Fig. 4C and D).

Effect of the combination of atorvastatin and BBR on foam cell formation. MCP-1 is a potent chemoattractant for monocytes and plays pivotal roles in the initiation and development of atherosclerosis by promoting monocyte infiltration to lesion-prone areas and penetration between endothelial cells into the inner arterial space $(23,24)$. BBR has been used as an anti-inflammatory agent. It has been reported that BBR inhibits the expression of MCP-1 in macrophages (25). Atorvastatin is a traditional anti-atheroscle- rotic drug. The anti-atherosclerotic effects of atorvastatin may be partly achieved by inhibiting the secretion of MCP-1 and the expression of SIRT1 in foam cells $(26,27)$.

In the present study, we investigated the anti-atherosclerotic effects of a combination of atorvastatin and BBR. As shown in Fig. 5, atorvastatin and BBR both suppressed the LPS-induced expression and secretion of MCP-1, as well as lipid accumulation. In addition, both atorvastatin and BBR upregulated the expression of SIRT1. However, the combination of atorvastatin and BBR was more effective than using atorvastatin alone. These data suggest a strong synergistic benefit of combination therapy with BBR and atorvastatin for preventing atherosclerotic processes.

\section{Discussion}

Atherosclerosis is a well-known multigenic, progressive chronic inflammatory disease. A characteristic of the atherosclerotic lesion is that macrophages change into foam cells after phagocytizing cholesterol during the development of atherosclerosis (28). The accumulation of lipid-laden foam cells is a critical step in the progression of atherosclerosis due to the augmented inflammation and impaired cholesterol metabolism within vascular walls $(6,29,30)$. 

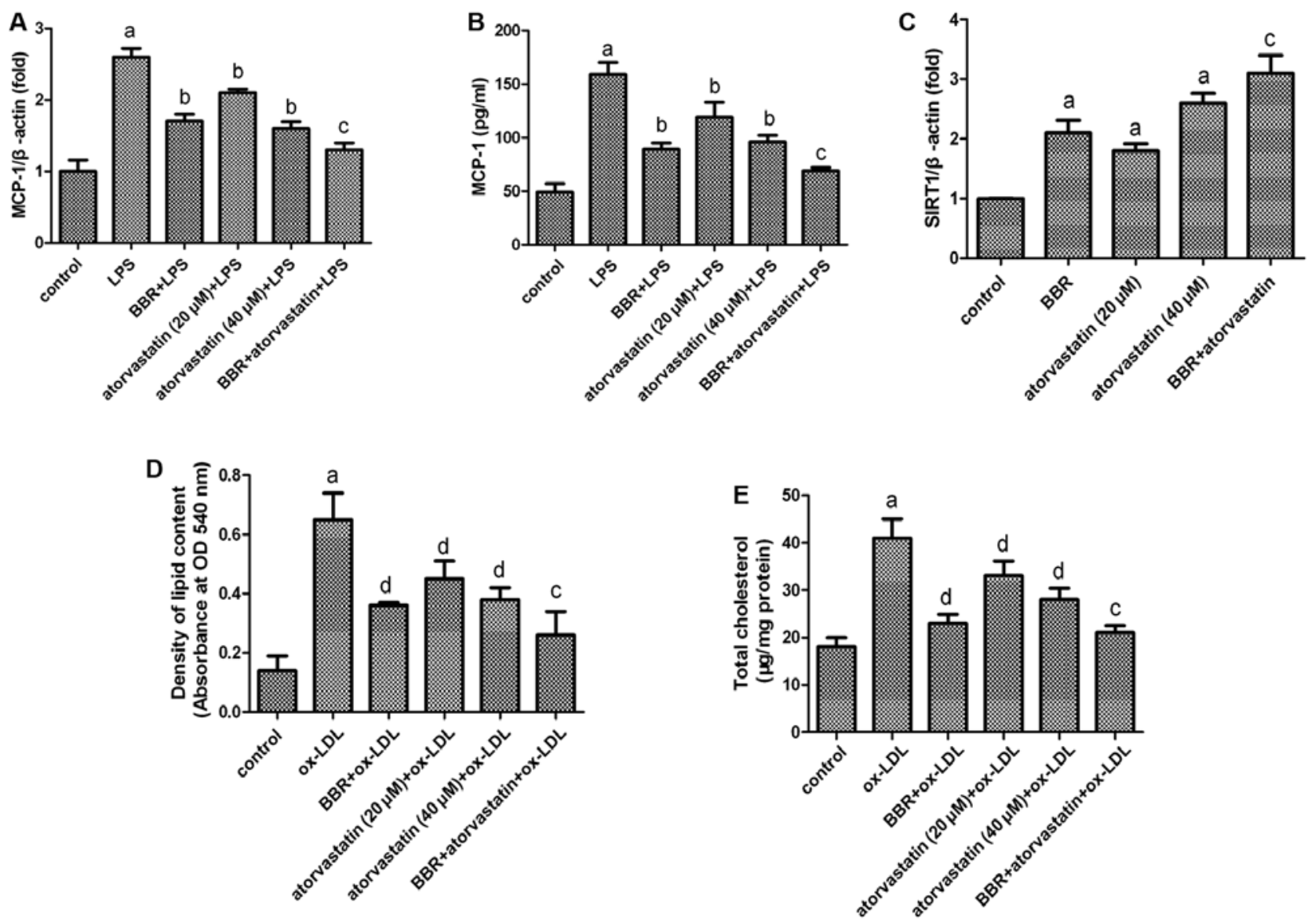

Figure 5. Effect of a combination of atorvastatin and berberine (BBR) on foam cell formation. THP-1-derived macrophages were divided into an atorvastatin (20 and $40 \mu \mathrm{M}), \mathrm{BBR}(10 \mathrm{mg} / \mathrm{l})$ and atorvastatin, or a combination of BBR plus atorvastatin groups ( $20 \mu \mathrm{M}$ atorvastatin, $10 \mathrm{mg} / \mathrm{l} \mathrm{BBR})$. The treatment groups were co-cultured with the drugs for $2 \mathrm{~h}$ and were then treated with oxidized low density lipoprotein (ox-LDL; $50 \mathrm{mg} / \mathrm{l}$ ) or lipopolysaccharide (LPS; $10 \mu \mathrm{M}$ ) for $12 \mathrm{~h}$ or were only treated with drugs for $24 \mathrm{~h}$. (A and B) Levels of monocyte chemotactic protein-1 (MCP-1); (C) mRNA level of sirtuin 1 (SIRT1); (D) density of lipid content; (E) cholesterol ester was assayed by HPLC. Data are the means \pm SD from 6 independent experiments. ${ }^{a}<<0.05$ vs. control (untreated cells); ${ }^{\mathrm{b}} \mathrm{P}<0.05$ vs. treatment with LPS; ${ }^{\mathrm{C}} \mathrm{P}<0.05$ vs. treatment with atorvastatin $(40 \mu \mathrm{M})$; ${ }^{\mathrm{d}} \mathrm{P}<0.05$ vs. treatment with ox-LDL.

BBR is a traditional anti-inflammatory medicine used in China. It has been shown to decrease the levels of LDL cholesterol (LDL-C), prevent oxidative stress and inhibit the migration and proliferation of vascular smooth muscle cells (13,31-36). Therefore, BBR has anti-atherosclerotic potency. In the present study, BBR significantly reduced the accumulation of lipids and cholesterol in THP-1-derived macrophages and suppressed foam cell formation. These results are consistent with those of previous studies demonstrating that treatment with BBR reduces serum cholesterol levels and impedes the development of atherosclerosis (13). According to this observation, we further elucidated the possible mechanisms underlying the BBR-mediated inhibition of foam cell formation.

During the formation of foam cells, the intake of cholesterol and reserve cholesterol transport play vital roles. It has been confirmed that the intake of cholesterol is mediated by certain proteins, such as PPAR- $\gamma$ and SIRT1. SIRT1 is one of the genes upstream of PPAR- $\gamma$. Our data demonstrated that treatment with BBR upregulated the expression of SIRT1 at both the mRNA and protein level and inhibited the expression of PPAR- $\gamma$. SIRT1 siRNA reversed the effects of BBR on the expression of PPAR- $\gamma$ and the accumulation of lipid and cholesterol in THP-1-derived macrophages. To investigate the mechanisms responsible for the effects of BBR on SIRT1, we evaluated the activation of AMPK, a regulator of SIRT1, which is an important serine/threonine kinase well known for regulating cellular energy levels by balancing nutrient availability and energy demand through its control of several proteins involved in glucose and lipid metabolism $(19,20)$. More importantly, we additionally demonstrated that BBR promoted the activation of AMPK, and compound $\mathrm{C}$, a specific inhibitor of AMPK reversed the effects of BBR on the expression of SIRT1 and PPAR- $\gamma$ and the accumulation of lipid and cholesterol in THP-1-derived macrophages.

It has been reported that atorvastatin exerts pleiotropic effects in patients with atherosclerosis through the inhibition of coronary atheroma progression, reducing clinical events and by increasing the expression of genes involved in the apoptosis of monocytes (37). Furthermore, we investigated the effects of treatment with BBR or atorvastatin alone, or a combination of both on foam cell formation. We found that atorvastatin and BBR both suppressed the LPS-induced expression and secretion of MCP-1 and lipid accumulation, and upregulated the expression of SIRT1. However, the combination of atorvastatin and BBR was more effective in than using atorvastatin alone. These data suggest a strong synergistic benefit of combination therapy with BBR and atorvastatin for preventing atherosclerotic processes. 
In conclusion, our results reveal a novel mechanism through which BBR prevents atherogenesis: BBR suppresses foam cell formation by activating the AMPK-SIRT1-PPAR- $\gamma$ pathway and diminishing the uptake of ox-LDL. Our findings provide a novel explanation for the anti-atherosclerotic activity of BBR and suggest that BBR may be a useful agent for the treatment of atherosclerosis. Furthermore, the results obtained in the present study demonstrate that a combination of atorvastatin and BBR is more effective than atorvastatin alone in inhibiting foam cell formation. Our data suggest a strong synergistic benefit of combination therapy with BBR and atorvastatin for preventing atherosclerotic processes.

\section{Acknowledgements}

The present study was supported by grants from the Foundation of Shaanxi Province Natural Science Research Project (2012JM4004) and the Foundation of Lanzhou Military Region Medical Scientific Research Project (CLZ12JA24).

\section{References}

1. Zhao JF, Jim Leu SJ, Shyue SK, Su KH, Wei J and Lee TS: Novel effect of paeonol on the formation of foam cells: promotion of LXR $\alpha$-ABCA1-dependent cholesterol efflux in macrophages. Am J Chin Med 41: 1079-1096, 2013.

2. Hansson GK: Inflammation, atherosclerosis, and coronary artery disease. N Engl J Med 352: 1685-1695, 2005.

3. Weber C, Zernecke A and Libby P: The multifaceted contributions of leukocyte subsets to atherosclerosis: lessons from mouse models. Nat Rev Immunol 8: 802-815, 2008.

4. Boring L, Gosling J, Cleary M and Charo IF: Decreased lesion formation in CCR $2^{--}$mice reveals a role for chemokines in the initiation of atherosclerosis. Nature 394: 894-897, 1998.

5. Edfeldt K, Swedenborg J, Hansson GK and Yan ZQ: Expression of toll-like receptors in human atherosclerotic lesions: a possible pathway for plaque activation. Circulation 105: 1158-1161, 2002.

6. Li AC and Glass CK: The macrophage foam cell as a target for therapeutic intervention. Nat Med 8: 1235-1242, 2002.

7. Voloshyna I, Hai O, Littlefield MJ, Carsons S and Reiss AB: Resveratrol mediates anti-atherogenic effects on cholesterol flux in human macrophages and endothelium via PPAR $\gamma$ and adenosine. Eur J Pharmacol 698: 299-309, 2013.

8. Stein S, Lohmann C, Schafer N, et al: SIRT1 decreases Lox-1mediated foam cell formation in atherogenesis. Eur Heart J 31: 2301-2309, 2010.

9. Picard F, Kurtev M, Chung N, et al: Sirt1 promotes fat mobilization in white adipocytes by repressing PPAR- $\gamma$. Nature 429: 771-776, 2004.

10. Vaziri H, Dessain SK, Ng Eaton E, et al: hSIR2(SIRT1) functions as an NAD-dependent p53 deacetylase. Cell 107: 149-159, 2001.

11. Zhang QJ, Wang Z, Chen HZ, et al: Endothelium-specific overexpression of class III deacetylase SIRT1 decreases atherosclerosis in apolipoprotein E-deficient mice. Cardiovasc Res 80: 191-199, 2008.

12. Huang Z, Dong F, Li S, et al: Berberine-induced inhibition of adipocyte enhancer-binding protein 1 attenuates oxidized lowdensity lipoprotein accumulation and foam cell formation in phorbol 12-myristate 13-acetate-induced macrophages. Eur J Pharmacol 690: 164-169, 2012.

13. Kong W, Wei J, Abidi P, et al: Berberine is a novel cholesterollowering drug working through a unique mechanism distinct from statins. Nat Med 10: 1344-1351, 2004.

14. Yin J, Xing $\mathrm{H}$ and Ye J: Efficacy of berberine in patients with type 2 diabetes mellitus. Metabolism 57: 712-717, 2008.

15. Liu X, Li G, Zhu H, et al: Beneficial effect of berberine on hepatic insulin resistance in diabetic hamsters possibly involves in SREBPs, LXR $\alpha$ and PPAR $\alpha$ transcriptional programs. Endocr J 57: 881-893, 2010.
16. Zhu X, Guo X, Mao G, et al: Hepatoprotection of berberine against hydrogen peroxide-induced apoptosis by upregulation of Sirtuin 1. Phytother Res 27: 417-421, 2013.

17. Guan $\mathrm{S}$ and Wang B: Effects of fosinopril and valsartan on expressions of ICAM-1 and NO in human umbilical vein endothelial cells. Chin Med J (Engl) 116: 923-927, 2003.

18. Winnik S, Stein S and Matter CM: SIRT1 - an anti-inflammatory pathway at the crossroads between metabolic disease and atherosclerosis. Curr Vasc Pharmacol 10: 693-696, 2012.

19. Vingtdeux V, Chandakkar P, Zhao H, Davies P and Marambaud P: Small-molecule activators of AMP-activated protein kinase (AMPK), RSVA314 and RSVA405, inhibit adipogenesis. Mol Med 17: 1022-1030, 2011.

20. Fullerton MD, Steinberg GR and Schertzer JD: Immunometabolism of AMPK in insulin resistance and atherosclerosis. Mol Cell Endocrinol 366: 224-234, 2013.

21. Canto C, Jiang LQ, Deshmukh AS, et al: Interdependence of AMPK and SIRT1 for metabolic adaptation to fasting and exercise in skeletal muscle. Cell Metab 11: 213-219, 2010.

22. Hardie DG: AMP-activated/SNF1 protein kinases: conserved guardians of cellular energy. Nat Rev Mol Cell Biol 8: 774-785, 2007.

23. Charo IF and Taubman MB: Chemokines in the pathogenesis of vascular disease. Circ Res 95: 858-866, 2004.

24. McLaren JE, Michael DR, Ashlin TG and Ramji DP: Cytokines, macrophage lipid metabolism and foam cells: implications for cardiovascular disease therapy. Prog Lipid Res 50: 331-347, 2011.

25. Chen FL, Yang ZH, Liu Y, et al: Berberine inhibits the expression of TNFa, MCP-1, and IL-6 in AcLDL-stimulated macrophages through PPAR $\gamma$ pathway. Endocrine 33: 331-337, 2008.

26. Zhu GY, Zhu XL, Li RT, Liu TB, Shang DY and Zhang Y: Atorvastatin inhibits scavenger receptor $A$ and monocyte chemoattractant protein-1 expressions in foam cell. Zhonghua Xin Xue Guan Bing Za Zhi 35: 666-669, 2007 (In Chinese).

27. Kawai H, Kurata T, Deguchi K, et al: Combination benefit of amlodipine plus atorvastatin treatment on carotid atherosclerosis in Zucker metabolic rats. Neurol Res 35: 181-186, 2013.

28. Guan S, Wang B, Li W, Guan J and Fang X: Effects of berberine on expression of LOX-1 and SR-BI in human macrophage-derived foam cells induced by ox-LDL. Am J Chin Med 38: 1161-1169, 2010.

29. Kunjathoor VV, Febbraio M, Podrez EA, et al: Scavenger receptors class A-I/II and CD36 are the principal receptors responsible for the uptake of modified low density lipoprotein leading to lipid loading in macrophages. J Biol Chem 277: 49982-49988, 2002.

30. Rader DJ and Pure E: Lipoproteins, macrophage function, and atherosclerosis: beyond the foam cell? Cell Metab 1: 223-230, 2005.

31. Abidi P, Zhou Y, Jiang JD and Liu J: Extracellular signalregulated kinase-dependent stabilization of hepatic low-density lipoprotein receptor mRNA by herbal medicine berberine. Arterioscler Thromb Vasc Biol 25: 2170-2176, 2005.

32. Brusq JM, Ancellin N, Grondin P, et al: Inhibition of lipid synthesis through activation of AMP kinase: an additional mechanism for the hypolipidemic effects of berberine. J Lipid Res 47: 1281-1288, 2006.

33. Li L, Sawamura T and Renier G: Glucose enhances human macrophage LOX-1 expression: role for LOX-1 in glucoseinduced macrophage foam cell formation. Circ Res 94: 892-901, 2004.

34. Liang KW, Ting CT, Yin SC, et al: Berberine suppresses MEK/ ERK-dependent Egr-1 signaling pathway and inhibits vascular smooth muscle cell regrowth after in vitro mechanical injury. Biochem Pharmacol 71: 806-817, 2006.

35. Yokozawa T, Ishida A, Kashiwada Y, Cho EJ, Kim HY and Ikeshiro Y: Coptidis Rhizoma: protective effects against peroxynitrite-induced oxidative damage and elucidation of its active components. J Pharm Pharmacol 56: 547-556, 2004.

36. Cho BJ, Im EK, Kwon JH, et al: Berberine inhibits the production of lysophosphatidylcholine-induced reactive oxygen species and the ERK1/2 pathway in vascular smooth muscle cells. Mol Cells 20: 429-434, 2005.

37. Wang ZH, Liu XL, Zhong M, et al: Pleiotropic effects of atorvastatin on monocytes in atherosclerotic patients. J Clin Pharmacol 50: 311-319, 2010. 